\title{
FEASIBILITY OF CRYOPRESERVED TRACHEAL XENOTRANSPLANTS WITH THE USE OF SHORT-COURSE IMMUNOSUPPRESSION
}

Mitsunori Hashimoto, MD

Ryoichi Nakanishi, MD

Masayoshi Umesue, MD

Hiroyuki Muranaka, MD

Mitsuhiro Hachida, MD

Kosei Yasumoto, MD
Objective: We evaluated the feasibility of discordant xenotransplantation of the cryopreserved trachea with intermittent immunosuppression to help solve the shortage of donor tracheas.

Methods: Two experiments were performed with heterotopic transplantation models in 14 guinea pigs and 85 rats. So that the minimal dose of FK506 for viable fresh xenografts could be determined, FK506 was given in escalating doses $(0,1.5,2.5$, and $3.5 \mathrm{mg} / \mathrm{kg})$ for recipient animals after xenogeneic transplantation. With the goal of obtaining a long-term survival of the xenografts, the effect of cryopreservation on xenografts was assessed and thereafter different cycles of immunosuppression every third week were evaluated in fresh or cryopreserved xenografts in the second experiment.

Results: An FK506 dosage of more than $2.5 \mathrm{mg} / \mathrm{kg}$ per day was much more effective than smaller dosages, as demonstrated by morphologic assessment. A higher dosage of FK506 potentially delayed the rejection of xenografts and can thus maintain tracheal xenograft viability for less than 4 weeks in rat recipients. In experiment 2 , the cryopreserved xenografts showed less histologic viability than fresh xenografts but greater patency of the lumen. The patency of cryopreserved xenografts was favorably maintained for a longer period than that of fresh xenografts with either the same number or more cycles of immunosuppression.

Conclusions: We conclude that the synergistic effect of cryopreservation and adequate intermittent immunosuppression may enable tracheal xenografts to remain viable over longer periods. (J Thorac Cardiovasc Surg 2001;121:241-8)
A long segment of the trachea is often affected by malignant diseases or by benign stenoses of the airway caused by a traumatic, congenital, inflammatory, or iatrogenic lesion. Because of the current lack of suit-

From the Second Department of Surgery, School of Medicine, University of Occupational and Environmental Health, Kitakyushu, Japan.

This study was supported by a Research Grant for Immunology, Allergy and Organ Transplant, Ministry of Health and Welfare, and Grant-in-Aid 08671555 from the Ministry of Education, Science, and Culture of Japan.

Received for publication April 17, 2000; revisions requested July 21, 2000; revisions received Aug 31, 2000; accepted for publication Sept 29, 2000.

Address for reprints: Ryoichi Nakanishi, MD, Assistant Professor, Second Department of Surgery, School of Medicine, University of Occupational and Environmental Health, 1-1 Iseigaoka, Yahatanishiku, Kitakyushu 807-8555, Japan (E-mail: ryoichi@med.uoeh-u.ac.jp).

Copyright (c) 2001 by The American Association for Thoracic Surgery

$0022-5223 / 2001 \$ 35.00+0 \quad \mathbf{1 2 / 1 / 1 1 2 2 0 6}$

doi: $10.1067 / \mathrm{mtc} .2001 .112206$ able replacement strategies for such diseased trachea, the research into tracheal transplantation is of importance. We previously studied graft viability, ${ }^{1,2}$ rejection reaction, ${ }^{3}$ minimal immunosuppression, ${ }^{4,5}$ and the reconstruction range $e^{2,6-9}$ in tracheal allotransplantation for eventual clinical use in human beings and obtained satisfactory results. The shortage of donor trachea remains a major obstacle, although transplantation of the trachea is considered to be the preferred method for the reconstruction of extensive tracheal defects in the future. Previously, we investigated the maximal period of cryopreservation for viable tracheal isografts to help solve this problem. ${ }^{10}$ As a result, cryopreservation of the trachea has been recognized to induce the degeneration of both the cartilage and epithelium. The permissible period of cryopreservation for tracheal isografts is considered to be 3 months because the cartilage showed irreversible damage after that point. ${ }^{10}$ We also investigated the limits of warm ischemia time before 3-month cryopreservation for viable tracheal isografts and found this period to be 18 hours in rats (unpublished data). 
These periods of preservation could therefore help us to solve the severe shortage of human tracheal tissue.

Xenogeneic cryopreserved tracheas are an appealing alternative to tracheal allografts for transplantation because of the potentially unlimited supply of tracheas for use in human beings. Guinea pig tracheal xenografts in rats have been the subject of relatively few studies. Earlier, we observed that a short course of immunosuppression with FK506 significantly retains the viability of rat tracheal allograft. ${ }^{11}$ Several investigators have also reported cryopreservation to reduce the tracheal antigenicity. ${ }^{12,13}$ We thus tried to elucidate the feasibility of the discordant xenotransplantation of cryopreserved tracheas using a short course of immunosuppression. The use of cryopreserved tracheal xenografts could directly help to solve the shortage of donors.

\section{Materials and methods}

Animals, anesthesia, and harvesting of tracheas. Fourteen male guinea pigs and 85 male Brown Norway rats between 10 and 12 weeks of age and weighing approximately 200 to $250 \mathrm{~g}$ were all premedicated with anesthetic ether. The animals were placed in the supine position and anesthetized with the intraperitoneal administration of sodium pentobarbital $(10 \mathrm{mg} / \mathrm{kg})$. Either harvesting or transplantation was performed with the animals breathing spontaneously without an endotracheal tube. The 14 donor guinea pigs and the 1 donor Brown Norway rat were put to death with an intraperitoneal overdose of pentobarbital and were left at room temperature $\left(24^{\circ} \mathrm{C}\right)$. After the donor animals were killed, the whole trachea was identified through a midline cervical to sternal incision and excised in continuity. Thereafter, the wound was closed in the usual fashion. The harvested trachea was trimmed off in 5ring segments. As a result, 5 or 6 tracheal segments consisting of 5 rings were harvested from each donor animal. All animals received humane care in compliance with the "Guide for the Care and Use of Laboratory Animals" published by the National Institutes of Health (NIH Publication No. 85-23, revised 1985).

Cryopreservation and grafting. The tracheal segments were then either used as fresh grafts or immediately stored in a plastic sterile tube filled with the freezing solution. The preservative solution in which the grafts were immersed contained a balanced buffered salt solution with L-glutamine (RPMI1640 medium; JRM Biosciences, Lenexa, Kan) with a final concentration of $20 \%$ fetal calf serum and $10 \%$ dimethyl sulfoxide as the cryoprotectant. The plastic tube containing the specimen was then stored in a Bicell biofreezing vessel (Nihon Freezer Co, Ltd, Tokyo, Japan) after cooling at $4{ }^{\circ} \mathrm{C}$ for an hour and was subsequently stored in a deep freezer at $-80^{\circ} \mathrm{C}$. The Bicell biofreezing vessel has the ability to cool at a rate of approximately $1^{\circ} \mathrm{C}$ per minute in a deep freezer until reaching $-80^{\circ} \mathrm{C} .{ }^{10}$ Cryopreserved xenografts were thawed in an incubator for 15 minutes at $37^{\circ} \mathrm{C}$ for grafting. All xenografts were stented over a silicone rod measuring 1.35 $\mathrm{mm}$ in diameter (ATOM, Inc, Tokyo, Japan). A silicone rod was used to prevent the omentum from intruding into the graft. A modified version of the heterotopic tracheal transplant model with omental wrapping has been used for rats because this model showed the same healing course as an orthotopic transplantation model in our previous experiments. ${ }^{3,5,10}$ The recipient animals were placed in a supine position. Through a small upper midline laparotomy, the greater omentum was delivered into the wound. The anterior layer of the omentum was opened, and the tracheal transplant was then completely enveloped. The omentum and the enclosed transplant were then returned to the peritoneal cavity, and the wound was closed.

Experimental design. Two experiments were performed to assess the utility of the cryopreserved xenogeneic trachea. The minimal dose of FK506 for viable fresh xenografts was determined by gradually increasing administrations of FK506 for the recipient animals in the first experiment. Different doses of FK506 were thus administered intramuscularly for only 3 consecutive days after heterotopic tracheal xenotransplantation. Syngeneic transplantation without any other treatment was performed in 4 rats and the isografts were evaluated 4 weeks after surgery (group 1). The remaining 48 animals that underwent a xenogeneic transplantation were randomly allocated into 4 treatment groups as follows: group $2(n=12)$, no immunosuppression; group $3(\mathrm{n}=12), 1.5 \mathrm{mg} / \mathrm{kg}$ dose of FK506; group $4(\mathrm{n}=12), 2.5 \mathrm{mg} / \mathrm{kg}$ dose of FK506; and group $5(\mathrm{n}=12), 3.5 \mathrm{mg} / \mathrm{kg}$ dose of FK506. To histologically observe the xenogeneic immune response and also to determine the minimal dose of FK506 for viable tracheal xenografts, we performed a second laparotomy to retrieve the transplants 1,2 , or 4 weeks after surgery, thereby creating subgroups of a, b, and c, respectively (Fig 1, $A$ ).

With the aim of achieving long-term xenograft survival, different cycles of immunosuppression every third week were evaluated with fresh or cryopreserved xenografts used in the second experiment. One cycle of immunosuppression was designed to administer a minimal dose of FK506, as determined in experiment 1 , for 3 consecutive days. The donor grafts were randomly assigned to 5 groups. Fresh (group 6: $\mathrm{n}$ $=8$ ) and cryopreserved (group 7: $\mathrm{n}=8$ ) xenografts that had both undergone 1 cycle of immunosuppression were evaluated 3 weeks after surgery, and the effect of cryopreservation on the xenograft was assessed. Two (group 8: $\mathrm{n}=8$ ) and 3 (group 9: $n=4$ ) cycles of the treatment for fresh xenografts and 2 cycles of treatment for cryopreserved xenografts (group 10: $n$ $=4$ ) were performed after transplantation to evaluate the longterm survival of the xenograft. The viability of the transplants was evaluated 8 weeks after transplantation in groups 8 and 9 and 11 weeks after surgery in group 10 (Fig 1, B).

Morphologic assessment. All tissue specimens were fixed in $10 \%$ formalin. Microscopic slides were made from cross sections of the midportion of the tracheal segment and adherent omentum and then were routinely stained with hematoxylin and eosin. Thereafter, all specimens were examined by light microscopy. We attempted to quantify the viability of 
the heterotopically grafted trachea by objectively calculating the percent patency of the cross-sectional area (CSA) of the middle part in the graft and subjectively evaluating the morphology of epithelium and cartilage. All assessments were performed in a blinded fashion.

Patency. The graft percent patency was expressed as a proportion of the CSA of the xenograft on the observed day after surgery to the same graft before surgery. The CSA was calculated by the following formula: CSA $=a / 2 \times b / 2 \times \pi$, where " $a$ " was the transverse diameter and " $b$ " was the sagittal diameter. ${ }^{2,4}$

Epithelial viability. Epithelial viability was evaluated according to the following grading system: $0=$ no epithelium, 1 = single-layer nonciliated epithelium, 2 = multilayer nonciliated epithelium, and 3 = normal mucociliary epithelium. The epithelium of the grafts was assessed as a ratio of the epithelial score on a microscopic slide. ${ }^{3,5,10}$

Cartilage viability. In experiment 1 , the viability of the cartilage of each transplant was graded semiquantitatively from 0 to 3 , with 0 indicating severe changes (necrotic area $>70 \%$ of a microscopic visual field), 1 indicating moderate changes $(30 \%<$ necrotic area $<70 \%), 2$ indicating mild changes (necrotic area $<30 \%$ ), and 3 indicating the absence of any abnormalities. ${ }^{3,5,6}$ More detailed data were investigated in experiment 2 . The ratio of chondrocytes possessing viable nuclei in the cartilage of each transplant was calculated after carefully counting the number of all chondrocytes in each high-power field on a microscopic slide. A viable chondrocyte nucleus was regarded as richly stained and possessing a clear nucleus membrane. ${ }^{10}$ The score of cartilage dislocation was then graded semiquantitatively from 0 to 3 , with 0 indicating the absence of any abnormalities, 1 indicating mild changes (incidence of cartilage dislocation $<30 \%$ of a microscopic visual field), 2 indicating moderate changes $(30 \%<$ incidence of dislocation $<70 \%$ ), and 3 indicating severe changes (incidence of dislocation $>70 \%$ ) in experiment 2 .

Statistical analysis. All data were presented as the mean \pm standard deviation of the mean. A statistical analysis was performed with the use of the paired Student $t$ test.

\section{Results}

Experiment 1. So that the minimal dose of FK506 for viable fresh xenografts could be determined, escalated doses of FK506 (0, 1.5, 2.5 and $3.5 \mathrm{mg} / \mathrm{kg})$ were administered to recipient animals after xenogeneic transplantation.

Animals. All animals survived the experimental protocol period. The weight of the recipient rats in group 5 decreased about 5\% until 2 weeks after surgery but had recovered to the control level by 4 weeks after the operation. No problem regarding weight was observed in other groups.

Macroscopic findings. All tracheal grafts were wrapped firmly by the omentum. Grossly, all free tracheal isografts appeared normal and also preserved their luminal rigidity, whereas the xenografts that
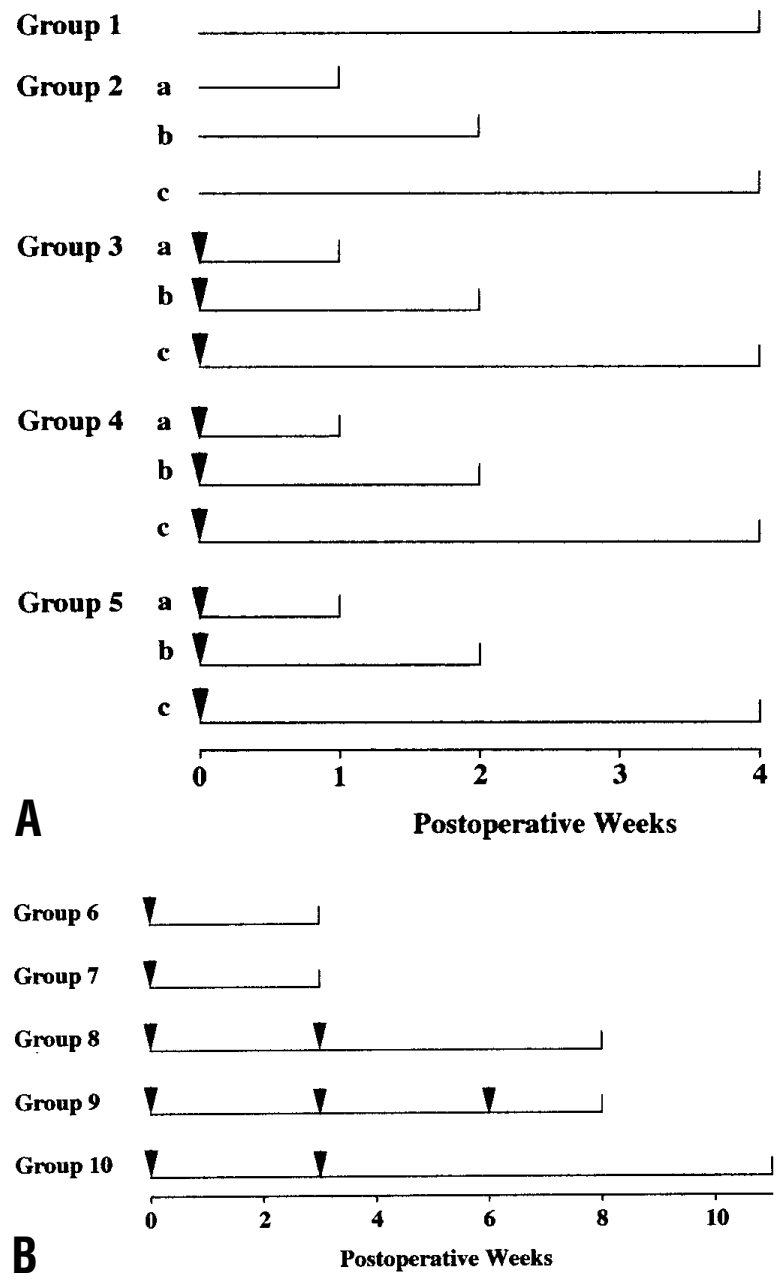

Fig 1. A, Design of experiment 1. B, Design of experiment 2. The arrowhead shows 1 cycle of immunosuppressive therapy with FK506 for 3 consecutive days.

underwent the lower dosage FK506 treatment did not demonstrate the same findings. The graft percent patency of groups $2 \mathrm{c}$ and $3 \mathrm{c}$ was significantly worse than that of the control group (Table I). The patency of groups $4 \mathrm{c}$ and $5 \mathrm{c}$ showed a better trend, according to a stepwise increase of dosage of FK506, than that of group 2c (group 2c vs $4 \mathrm{c}, P=.0592 ; 2 \mathrm{c}$ vs $5 \mathrm{c}, P=$ .0511 ) and did not demonstrate any significant difference from the control group (Fig 2). Mucoid fluid filled the lumen of almost all isografts in group 1 but not in groups 2 to 5 .

Microscopic findings. Both a lower dosage of FK506 and a long-term observation apparently resulted in an irreversible deterioration of the tracheal xenografts. The epithelium of the xenografts in groups 2 and 3 was almost denuded by 2 weeks after 
Table I. Percent patency of tracheal grafts in experiment 1

\begin{tabular}{lccc}
\hline Groups & Transplantation & Dosage of $F K 506(\mathrm{mg} / \mathrm{kg})$ & Patency $(\%)$ \\
\hline 1 (control) & Syngeneic & 0 & 100 \\
2c & Xenogeneic & 0 & $48.85 \pm 5.500^{*}$ \\
3c & Xenogeneic & 1.5 & $57.81 \pm 8.559^{*}$ \\
$4 \mathrm{c}$ & Xenogeneic & 2.5 & $80.40 \pm 19.95$ \\
$5 \mathrm{c}$ & Xenogeneic & 3.5 & $84.10 \pm 18.00$ \\
\hline
\end{tabular}

Data are presented as mean \pm standard deviation of the mean.

${ }^{*}$ The percent patency in each group was significantly worse than that in the control group (group 1 vs $2 \mathrm{c}, P=.0003 ; 1$ vs $3 \mathrm{c}, P=.0134$ ).

Table II. Comparison between fresh and cryopreserved tracheal xenografts 3 weeks after surgery

\begin{tabular}{|c|c|c|c|c|c|c|}
\hline Groups & $\begin{array}{l}\text { Graft } \\
\text { status }\end{array}$ & Patency (\%) & $\begin{array}{c}\text { Epithelial } \\
\text { score }\end{array}$ & $\begin{array}{c}\text { Viable } \\
\text { chondrocyte } \\
\text { ratio }\end{array}$ & $\begin{array}{c}\text { Cartilage } \\
\text { dislocation } \\
\text { score }\end{array}$ & $\begin{array}{c}\text { Mononuclear } \\
\text { cell infiltration } \\
\text { score }\end{array}$ \\
\hline 6 & Fresh & $65.59 \pm 21.98$ & $0.650 \pm 0.382$ & $0.313 \pm 0.139$ & $0.750 \pm 0.886$ & $1.500 \pm 1.195$ \\
\hline 7 & Cryop & $82.48 \pm 18.28$ & $0.325 \pm 0.437$ & $0.161 \pm 0.079^{*}$ & $0 \dagger$ & $0.750 \pm 0.463$ \\
\hline
\end{tabular}

Data are presented as mean \pm standard deviation of the mean. Cryop, Cryopreserved.

*The chondrocyte ratio in group 7 was significantly lower than that in group $6(P=.0098)$.

$\dagger$ The cartilage dislocation score in group 7 was significantly lower than that in group $6(P=.0479)$.

Table III. Morphologic features of tracheal xenografts receiving multiple cycles of immunosuppression

\begin{tabular}{|c|c|c|c|c|c|c|c|c|}
\hline Groups & $\begin{array}{l}\text { Graft } \\
\text { status }\end{array}$ & $\begin{array}{c}T x \\
\text { cycles }\end{array}$ & $\begin{array}{c}\text { Postop } \\
\text { weeks }\end{array}$ & $\begin{array}{c}\text { Patency } \\
(\%)\end{array}$ & $\begin{array}{c}\text { Epithelial } \\
\text { score }\end{array}$ & $\begin{array}{l}\text { Viable } \\
\text { chondrocyte } \\
\text { ratio }\end{array}$ & $\begin{array}{c}\text { Cartilage } \\
\text { dislocation } \\
\text { score }\end{array}$ & $\begin{array}{c}\text { Mononuclear } \\
\text { cell infiltration } \\
\text { score }\end{array}$ \\
\hline 8 & Fresh & 2 & 8 & $78.03 \pm 16.54$ & $0.025 \pm 0.071$ & $0.259 \pm 0.164$ & $0.250 \pm 0.463$ & $1.625 \pm 0.744$ \\
\hline 9 & Fresh & 3 & 8 & $84.36 \pm 18.85$ & 0 & $0.473 \pm 0.155$ & $0.125 \pm 0.354$ & 0 \\
\hline 10 & Cryop. & 2 & 11 & $85.98 \pm 17.85$ & $0.110 \pm 0.143$ & $0.317 \pm 0.128$ & $0.250 \pm 0.463$ & $0.750 \pm 0.500$ \\
\hline
\end{tabular}

Data are presented as mean \pm standard deviation of the mean. Tx, Treatment; Postop, postoperative; Cryop, cryopreserved.

the operation. The score in group 2 was significantly worse than that in groups 4 and 5 (groups $2 \mathrm{~b}$ vs $4 \mathrm{~b}$, $P=.0209 ; 2 \mathrm{a}$ vs $5 \mathrm{a}, P=.0009 ; 2 \mathrm{~b}$ vs $5 \mathrm{~b}, P=.0119$; $2 \mathrm{c}$ vs $5 \mathrm{c}, P=.0202$ ). In addition, each epithelial score in group 3 was significantly worse than that in groups 4 and 5 (groups $3 \mathrm{~b}$ vs $4 \mathrm{~b}, P=.0155 ; 3 \mathrm{a}$ vs $5 \mathrm{a}, P=$ .0225 ; 3 b vs $5 \mathrm{~b}, P=.0096 ; 3$ c vs 5 c, $P=.0202$ ) (Fig $3, A)$. In the interstitium, extensive hemorrhaging was seen in all groups 1 week after the operation. Submucosal thickening caused by fibrosis and necrosis gradually increased in the lower dosage FK506 groups by 4 weeks after surgery, whereas the same findings decreased in the higher dosage groups. Some xenografts of the higher dosage groups first showed a high infiltration of mononuclear cells 4 weeks after the operation. Regarding cartilage viability, the semiquantitative score in groups 2 and 3 was significantly worse than that in groups 4 and 5 (groups $2 \mathrm{~b}$ vs $4 \mathrm{~b}, P$ $=.0060 ; 2 \mathrm{~b}$ vs $5 \mathrm{~b}, P=.0138 ; 2 \mathrm{c}$ vs $4 \mathrm{c}, P=.0163 ; 2 \mathrm{c}$ vs 5 c, $P=.0032 ; 3$ c vs 5 c, $P=.0377$ ) (Fig $3, B$ ). According to the length of the observation period, the epithelial score showed a significant exacerbation in the subgroups of group 5 (groups $5 \mathrm{a}$ vs $5 \mathrm{c}, P=.0462$; $5 \mathrm{~b}$ v $5 \mathrm{c}, P=.0082)($ Fig $3, A)$, and the cartilage score showed a significant exacerbation in the subgroups of group 2 (groups $2 \mathrm{a}$ vs $2 \mathrm{~b}, P=.0060 ; 2 \mathrm{a}$ vs $2 \mathrm{c}, P=$ .0032) (Fig 3, B). Four weeks after surgery, the epithelial score in groups $2 \mathrm{c}, 3 \mathrm{c}$, and $5 \mathrm{c}$ was significantly worse than that of the control group (2.362 \pm $0.189)(P=.0001, .0004$, and .0033 , respectively), and the cartilage score in group $2 \mathrm{c}$ was significantly worse than that of the control group $(2.750 \pm 0.500)$ $(P=.0029)$. The xenografts administered FK506 in doses of 2.5 to $3.5 \mathrm{mg} / \mathrm{kg}$ (groups 4 and 5) showed a moderate grade of degeneration in the epithelium but possessed single-layer and/or multilayer nonciliated epithelium in some parts and low-grade degeneration of the cartilage 4 weeks after surgery (Fig 2). 


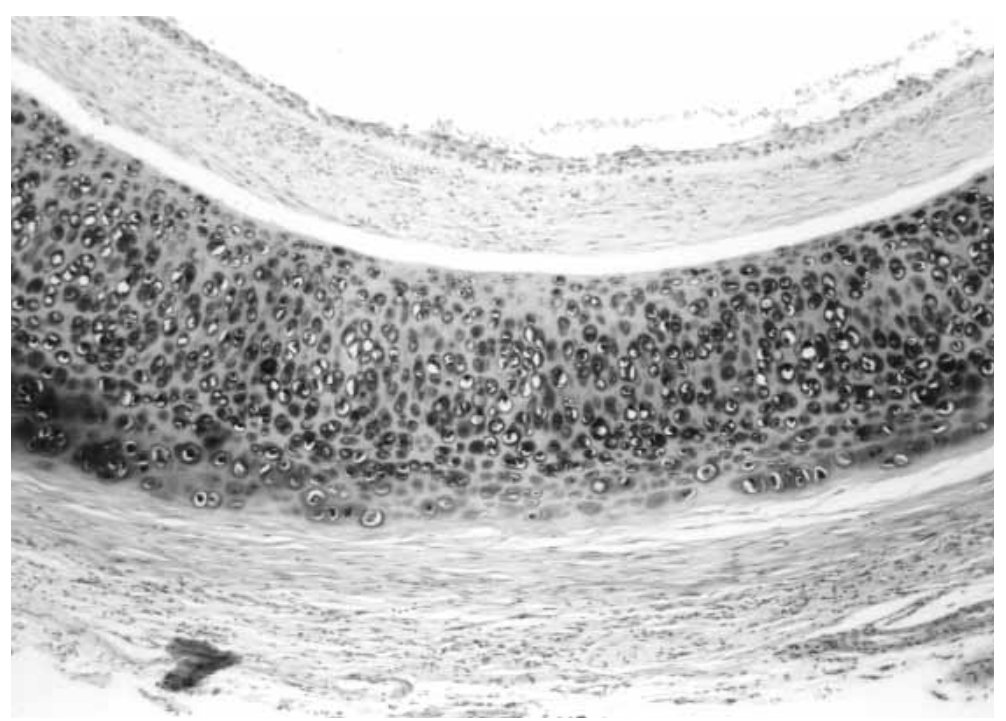

Fig 2. Histologic findings in fresh xenografts 4 weeks after surgery in group 5c. A single-layer and multilayer nonciliated epithelium is recognized. The cartilage has mostly viable chondrocyte nuclei. Mononuclear cell infiltration is only slightly seen. Submucosal thickening is seen, but the patency of the graft is well maintained. (Hematoxylin and eosin stain; original magnification, $\times 100$.)
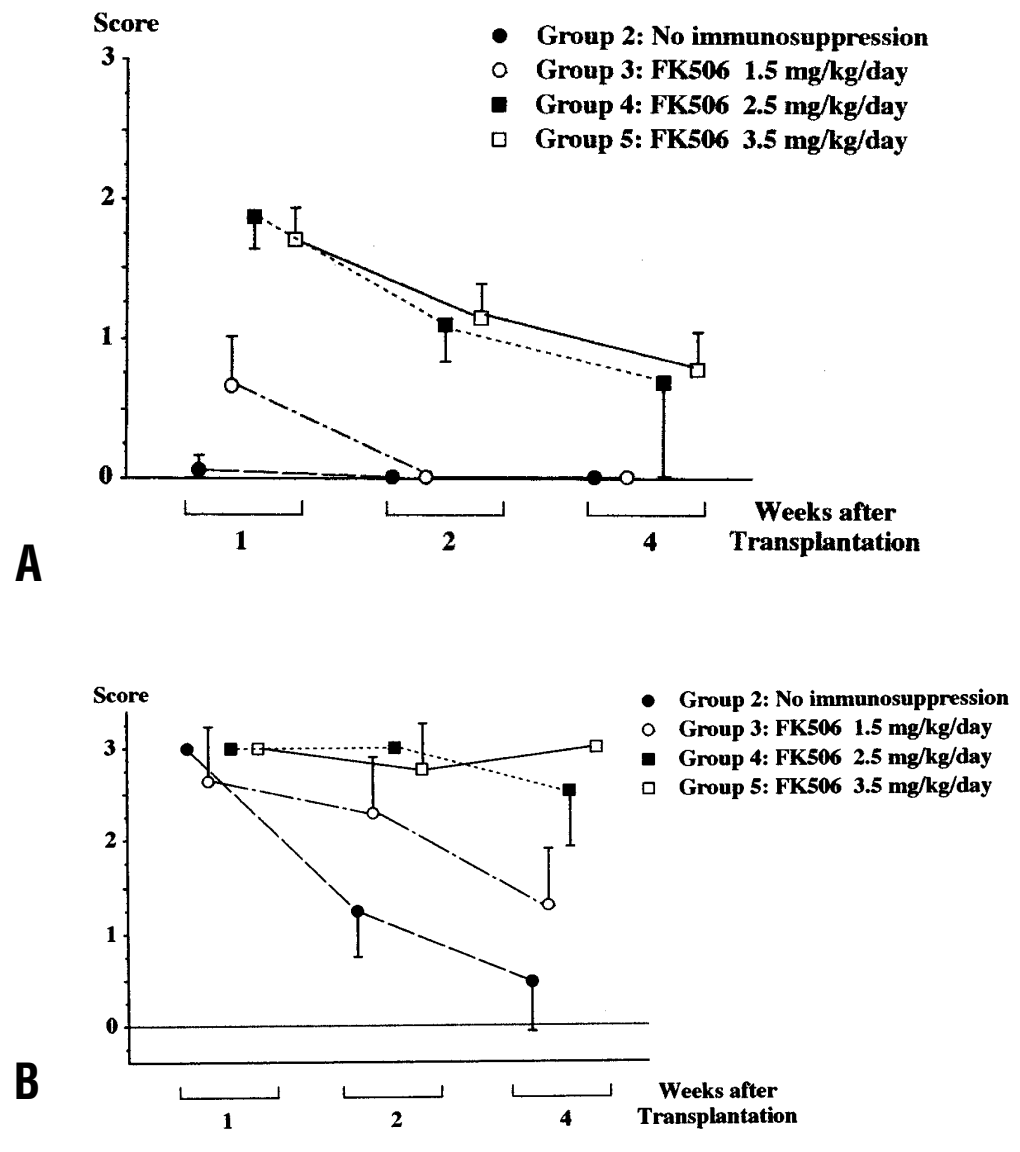

Fig 3. A, The epithelial score (mean \pm standard deviation of the mean) of tracheal xenografts in groups 2 to 5 . $\mathbf{B}$, The cartilage score (mean \pm standard deviation of the mean) of tracheal xenografts in groups 2 to 5 . 


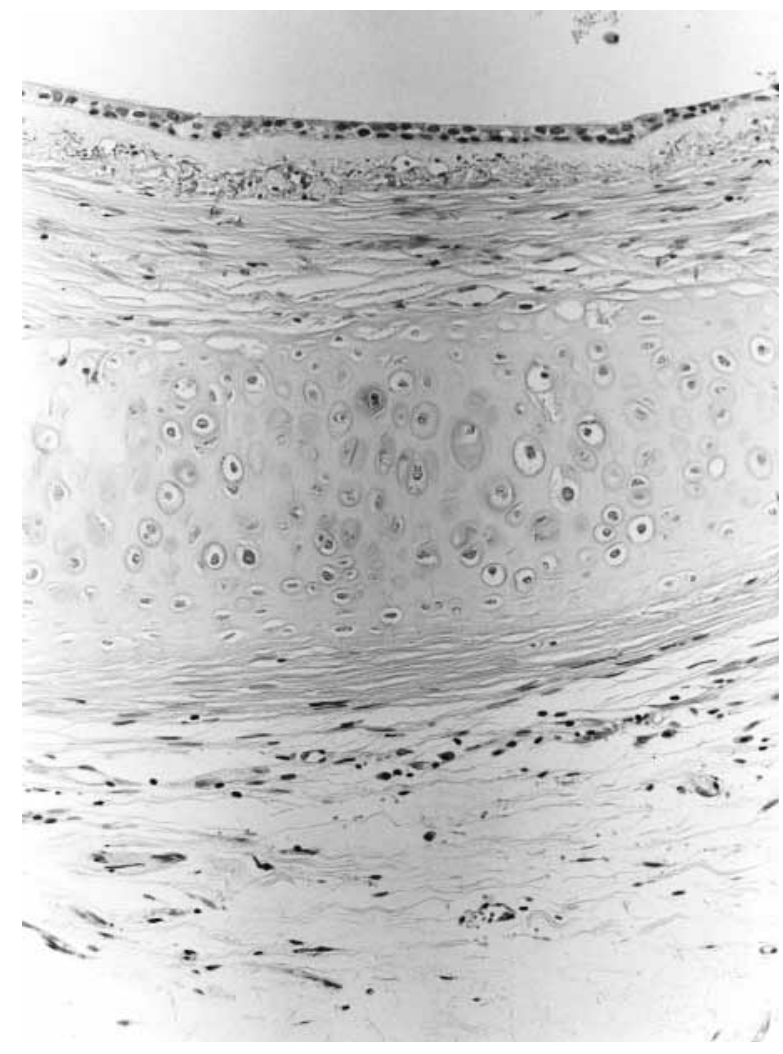

Fig 4. Histologic findings of cryopreserved xenografts 11 weeks after surgery in group 10 (undergoing 2 cycles of immunosuppression). A single-layer and multilayer nonciliated epithelium is extensively recognized. The cartilage has viable chondrocyte nuclei for the most part. Mononuclear cell infiltration is only slightly seen. Some submucosal thickening is seen but the patency of the graft is well maintained. (Hematoxylin and eosin; original magnification, $\times 200$.)

Experiment 2. With the goal of obtaining long-term survival of the xenografts, the effect of cryopreservation on xenografts was assessed and thereafter different cycles of immunosuppression every third week were evaluated in fresh or cryopreserved xenografts in the second experiment.

Animals. All animals received different cycles of immunosuppression consisting of an FK506 dose of $2.5 \mathrm{mg} / \mathrm{kg}$ determined in experiment 1 , and all survived for the entire experimental protocol period. No problem regarding the weight of any group was observed.

Macroscopic findings. The omentum was wrapped firmly around the tracheal xenografts in all rats. Grossly, cryopreserved tracheal xenografts in group 10 appeared to be almost normal and their luminal rigidity was preserved, whereas fresh xenografts in group 6 were swollen and the lumen showed a slight narrowing. The percent patency of the cryopreserved xenografts (group 7) was slightly better than that of the fresh xenografts (group 6) 3 weeks after surgery (Table II). Regarding the groups that were observed long term, the percent patency of the cryopreserved xenografts in group 10 was slightly higher than that of the fresh xenografts in group 8 (Table III). No mucoid fluid filled the lumen of any xenografts in groups 6 to 10 because the mucociliated epithelium and tracheal glands had been completely obliterated.

Microscopic findings. Three weeks after surgery, the viable chondrocyte ratio of the cryopreserved xenografts was significantly worse than that of the fresh grafts, and the same trend was also seen in the epithelial score. On the other hand, the cryopreserved xenografts showed a significantly better cartilage dislocation score than fresh grafts, and the same trend was also seen for the mononuclear cell infiltration score (Table II). In the groups observed long term, the histologic findings of the cryopreserved xenografts (group 10) were almost the same as those of the fresh xenografts (groups 8 and 9), despite a longer period of observation in group 10. Fresh xenografts showed moderate degeneration of the epithelium in group 6 and slightly higher degeneration in groups 8 and 9 , despite receiving multiple cycles of treatment. On the other hand, the cryopreserved xenografts showed some single-layer nonciliated epithelium, but none of them completely lost their epithelium despite prolonged observation (Fig 4). Submucosal thickening and mononuclear cell infiltration were often seen in the fresh xenografts of groups 6 and 8 . Viable tracheal glands were seen in some cryopreserved xenografts. Regarding the cartilage, no difference was seen in the viable chondrocyte ratio and the cartilage dislocation score between the fresh (groups 8 and 9) and cryopreserved (group 10) xenografts in the groups observed long term (Table III).

\section{Discussion}

The mechanism for host rejection of xenografts differs from that of allografts. Humoral responses play an important role in discordant xenograft rejection. Only immunosuppressive agents, therefore, must be incapable of reducing or delaying the rejection of xenografts. However, several investigators have already described that an appropriate immunosuppressive regimen can prolong xenograft survival. Anti-rat lymphocyte serum alone significantly prolonged xenograft functional survival, and no hyperacute rejection reaction occurred in a pig-to-rat xenotransplantation 
model. ${ }^{14,15}$ Valdivia and associates ${ }^{16}$ reported FK506 to potentially suppress antibody production in hamster-torat xenotransplantation. Hebebrand and colleagues ${ }^{17}$ showed FK506 to be a better immunosuppressive agent than RS61443, which they expected for xenograft survival because of its mechanism of action to reduce natural antibodies. For these reasons, we also selected FK506 alone as an immunosuppressant in these experiments. We designed these experiments using the same protocol of consecutive 3-day administrations of FK506 as we had previously used in rat allotransplantation models. ${ }^{11}$

A rapid rejection was seen in all rats that were not immunosuppressed in group 2, but a viable graft survival was achieved with adequate FK506 immunosuppression. A dosage of more than $2.5 \mathrm{mg} / \mathrm{kg}$ per day was much more effective than any smaller doses, as demonstrated by a morphologic assessment in experiment 1 . A higher dosage of FK506 may delay the rejection of xenografts and maintain tracheal xenograft viability for less than 4 weeks in rat recipients because a high infiltration of mononuclear cells was first seen in wellmaintained xenografts 4 weeks after surgery. We therefore designed the immunosuppression every third week for fresh or cryopreserved xenografts in the second experiment. The present findings indicate that the rejection of adult guinea pig xenografts in rats is sensitive to FK506, suggesting an important role for T cells in the rejection process. In addition, this dosage may be tolerable for the health of the recipient, as demonstrated by the minimal weight changes of animals.

Tze, Tai, and Chung ${ }^{14}$ reported that hyperacute rejection between discordant species does not occur in tissue xenotransplantation, probably because of low natural antibody titers. In general, cellular rejection is considered to be closely associated with tissue xenografts. There is also no firm evidence that any hyperacute rejection of tracheal xenografts occurred in our series. The detailed mechanism of tissue xenograft rejection remains unclear. Another mechanism of rejection added to the $\mathrm{T}$ cell-derived response may exist in tracheal xenotransplantation because the findings of xenograft rejection were slightly more severe than those of allograft rejection in our studies. ${ }^{3-5}$ A primitive immunoreaction may be associated with tracheal xenotransplantation, as evidenced by the effect of intensive immunosuppression. ${ }^{18}$

The epithelial morphology is reported to be closely associated with the rejection reaction in tracheal transplantation inasmuch as the epithelium has a strong antigenicity. ${ }^{19,20}$ The epithelium always disappears after heterotopic transplantation without immunosuppression. Thus, when the epithelium remains in tracheal allografts receiving immunosuppression, it means that immunosuppressive therapy has been effective. ${ }^{3-6}$ However, a donor epithelium is not necessarily needed in orthotopic tracheal transplantation because the donor epithelium is denuded after transplantation and the lumen of the graft is then covered with a migrated recipient epithelium. ${ }^{12}$ The assessment of the cartilage is more important than the assessment of the donor epithelium, which is considered to sensitively reflect graft viability in the heterotopic tracheal transplantation models, because the donor cartilage is not replaced by the recipient cartilage. ${ }^{3-6}$ We therefore studied in detail the donor cartilage, which plays an important role in keeping luminal rigidity. We also assessed the cartilage dislocation, which is closely associated with the percent patency. ${ }^{1-10}$ We gained seemingly conflicting results: for instance, the cryopreserved xenografts in group 7 showed less histologic viability than fresh xenografts in group 6, whereas the percent patency of their lumen indicated paradoxic findings. Cryopreserved xenografts did not show any cartilage dislocation despite a lower viability of chondrocyte. The cartilage dislocation is caused by the cellular infiltration and fibrosis and is not associated with the viable chondrocyte ratio. ${ }^{10}$ Cryopreservation has a degenerative effect on both the epithelium and cartilage and can potentially lead to a reduced antigenicity in both components. ${ }^{10,12,13}$ Perhaps the low antigenicity caused by cryopreservation may reduce cellular infiltration and consequently cartilage dislocation may not occur. The fact that the patency of cryopreserved xenografts 11 weeks after surgery was almost equivalent to that of fresh xenografts with either the same number of cycles or more immunosuppression 8 weeks after surgery, and was well maintained, may support our speculation.

We have thus shown that prolonged survival of discordant cryopreserved tracheal xenografts can be achieved with FK506 monotherapy. The synergistic effect of cryopreservation and adequate intermittent immunosuppressive therapy may play an important role in maintaining xenograft viability for long periods. Further studies are needed, however, to elucidate the mechanism of tracheal xenograft rejection, to maintain viable graft stability, and to investigate the usefulness of the potential source of xenografts, for example, porcine or primate, for clinical use in human beings.

We thank Mrs Miki Shimada for her skillful technical assistance. 


\section{REFERENCES}

1. Nakanishi R, Shirakusa T, Takachi T. Omentopexy for tracheal autografts. Ann Thorac Surg 1994;57:841-5.

2. Nakanishi R, Shirakusa T, Mitsudomi T. Maximum length of tracheal autografts in dogs. J Thorac Cardiovasc Surg 1993;106:1081-7.

3. Nakanishi R, Shirakusa T, Hanagiri T. Early histopathologic features of tracheal allotransplantation rejection: a study in nonimmunosuppressed dogs. Transplant Proc 1994;26:3715-8.

4. Nakanishi R, Yasumoto K, Shirakusa T. Short-course immunosuppression after tracheal allotransplantation in dogs. J Thorac Cardiovasc Surg 1995;109:910-7.

5. Nakanishi R, Yasumoto K. Minimal dose of cyclosporin A for tracheal allografts. Ann Thorac Surg 1995;60:635-9.

6. Takachi T, Shirakusa T, Nakanishi R, Okabayashi K, Inutsuka K, Kawahara K, et al. Experimental carinal autotransplantation and allotransplantation. J Thorac Cardiovasc Surg 1995;110:762-7.

7. Nakanishi R, Nagaya N, Yoshimatsu T, Hanagiri T, Yasumoto K. Optimal dose of basic fibroblast growth factor for long-segment orthotopic tracheal autografts. J Thorac Cardiovasc Surg 1997;113:26-36.

8. Nakanishi R, Hashimoto M, Yasumoto K. Improved airway healing using basic fibroblast growth factor in a canine tracheal autotransplantation model. Ann Surg 1998;227:446-54.

9. Nakanishi R, Hashimoto M, So T, Sugaya S, Yasumoto K. Successful tracheo-carinal transplantation. J Cardiovasc Surg 1999;40:591-6.

10. Nakanishi R, Hashimoto M, Muranaka H, Umesue M, Kohno H, Yasumoto K. Maximal period of cryopreservation using Bicell biofreezing vessel for rat tracheal isografts. J Thorac Cardiovasc Surg 1999;117:1070-6.
11. Hashimoto M, Nakanishi R, Muranaka H, Umesue M, Eifuku R, Yasumoto K. Short-course immunosuppression using FK506 for rat tracheal allografts. J Cardiovasc Surg 2000;41:487-92.

12. Tojo T, Kitamura S, Gojo S, Kushibe K, Nezu K, Taniguchi S. Epithelial regeneration and preservation of tracheal cartilage after tracheal replacement with cryopreserved allograft in the rat. J Thorac Cardiovasc Surg 1998;116:624-7.

13. Yokomise H, Inui K, Wada H, Hasegawa S, Ohno N, Hitomi S. Reliable cryopreservation of trachea for one month in a new trehalose solution. J Thorac Cardiovasc Surg 1995;110:382-5.

14. Tze WJ, Tai J, Cheung SSC. Transplantation of discordant pig islet xenografts in diabetic rats. Diabetes Res Clin Pract 1992; 15:197-204

15. Auchincloss H Jr. Xenogeneic transplantation. Transplant Proc 1988;46:1-20.

16. Valdivia LA, Demetris AJ, Fung JJ, Celli S, Murase N, Starzl TE. Successful hamster-to-rat liver xenotransplantation under FK506 immunosuppression induces unresponsiveness to hamster heart and skin. Transplantation 1993;55:659-61.

17. Hebebrand D, Jones NF, Zohman G, Rao U, Soleiman N. Limb xenotransplantation using FK506 and RS61443 immunosuppression. J Reconstr Microsurg 1998;14:191-4.

18. Blakely ML, Werf WJ, Berndt MC, Dalmasso AP, Bach FH, Hancock WW. Activation of intragraft endothelial and mononuclear cells during discordant xenograft rejection. Transplantation 1994;58:1059-66.

19. Lane BP, Habicht GS, Jasper GS. Lymphocyte-epithelium interaction during rejection of nonisogeneic rat tracheal grafts. Am J Pathol 1977;86:71-7.

20. Beigel A, Muller-Ruchholtz W. Tracheal transplantation. I. The immunogenic effect of rat tracheal transplants. Arch Otorhinolaryngol 1984;240:185-92.

\section{Online-www.aats.org}

Now you can get The Journal of Thoracic and Cardiovascular Surgery online.

The Journal online brings you faster delivery time, easy searching of current and back issues, links to PubMed, AATS, WTSA, and other important sites, and more. Visit the Journal online today.

\section{Receive tables of contents by e-mail}

To receive the tables of contents by e-mail, sign up through our Web site at http://www.mosby.com/jtcvs Choose E-mail Notification

Simply type your e-mail address in the box and click the Subscribe button.

Alternatively, you may send an e-mail message to majordomo@mosby.com. Leave the subject line blank and type the following as the body of your message: subscribe jtcvs_toc

You will receive an e-mail to confirm that you have been added to the mailing list. Note that TOC e-mails will be sent out when a new issue is posted to the Web site. 